\title{
The open circuit nitrogen washout technique for measuring the lung volume in infants: methodological aspects
}

\author{
Mohy G Morris
}

\begin{abstract}
Background-Lung volume measurement by nitrogen washout is widely used in infants, though a lack of accuracy and changes of calibration over time have been reported. The potential sources of error were explored in order to increase the accuracy and reliability of the technique. Methods-A commercial system for nitrogen washout and a 0.5 litre calibrating syringe as a lung model were used to perform over 2000 in vitro washouts, including simulated rapid breathing, shallow breathing, periodic breathing, sighs, and brief apnoeas. A constant $10 \mathrm{1} / \mathrm{min}$ bias flow of oxygen and extended equipment warming times were employed. A collapsible breathing bag was incorporated into the washout circuit. Following a single two point calibration, known air volumes from $42 \mathrm{ml}$ to $492 \mathrm{ml}$ were measured by nitrogen washout over a 14 hour period. The flow waveform in the nitrogen mixing chamber during a washout in vitro, with and without the breathing bag in the circuit, was also studied.
\end{abstract}

Results-The mean coefficient of variation of all volumes was $0.66 \%$. The mean difference between measured and known volumes was $0.30 \mathrm{ml}(95 \%$ confidence interval (CI) -0.18 to 0.79 ). This difference was not statistically significant $(p=0.22)$. The mean percentage error was $-0.1 \%$ (range $-0.47 \%$ to $0.46 \%$ ). Nitrogen calibration remained stable for 14 hours. Without the breathing bag flow transients were frequent in the mixing chamber during in vitro washout.

Conclusions-This technique increases the accuracy in vitro and the precision in vivo of volume measurement by nitrogen washout. Sources of potential errors including baseline drifting and inadequate equipment warming times were identified. The breathing bag acted as a buffer reservoir, preventing large swings in flows within the nitrogen mixing chamber during washouts, and should be an integral component of the nitrogen washout circuit.

(Thorax 1999;54:790-795)

Keywords: lung function tests; gas dilution; nitrogen washout; infants

Measurements of lung volumes in infants have been performed using whole body plethysmography and gas dilution. The former requires sophisticated equipment and a well trained operator and cannot generally be performed at the bedside or be used in sick infants. Gas dilution employs either helium or nitrogen as the tracer gas. The open circuit nitrogen washout technique is widely used because the dead space and circuit resistance are lower than in the helium closed circuit, making it suitable for small or sick infants. Moreover, data acquisition and calculation are easily programmed for a personal computer. ${ }^{1-5}$

While the measurement of lung volume has been regarded as physiologically and clinically important, ${ }^{12}$ the accuracy of the measurement is undoubtedly equally important. A commercial system has been used for the past 10 years or so in infant pulmonary function laboratories to measure the functional residual capacity (FRC) by nitrogen washout. ${ }^{4}$ Studies using this system have occasionally explored the accuracy of the nitrogen washout technique and possible sources of errors. ${ }^{5}$ A change of calibration over time was noted in a study on healthy infants and, despite efforts by the investigator to recheck calibration immediately prior to subject testing, a correction factor had to be used on several test results. ${ }^{5}$ Indeed, a recent study comparing lung volume measurements by whole body plethysmography and nitrogen washout reported a lack of accuracy and reliability of the two techniques in infants with airway obstruction and concluded that no "gold standard" technique was available for use in this setting. ${ }^{6}$

The primary aim of this investigation was therefore to enhance the accuracy of measurements and achieve a reliable calibration of the nitrogen washout technique by a systematic exploration of potential sources of errors in vitro using a calibrating syringe as a lung model, and ultimately to develop a method that renders volume measurements by nitrogen washout reliable and reproducible in vitro and in vivo. We also wanted to establish the linearity of the system over a wide range of volumes so that a single two point calibration could be employed to measure the functional residual capacity (FRC) and, using a newly developed method, the residual lung volume (RV) in infants. $^{7}$

\section{Methods}

A commercial system known as the Pediatric Pulmonary Unit (PPU) 2600 (SensorMedics, Anaheim, California, USA) was used. A 0.5 litre calibrating syringe (Hans Rudolph Inc, Kansas City, Missouri, USA) was employed as a lung model. 
NITROGEN WASHOUT TECHNIQUE

The open circuit nitrogen washout method for assessment of $\operatorname{FRC}\left(\mathrm{N}_{2}\right)$ as described by Gerhardt et al involves measuring the volume of nitrogen expired after end tidal expiratory switching of the inspired gas from room air to $100 \%$ oxygen. $\mathrm{RV}\left(\mathrm{N}_{2}\right)$ was also estimated after end forced expiratory switching. ${ }^{7}$ At a constant bias flow that exceeds the infant's inspiratory peak flow during tidal breathing, the integrated expired nitrogen is multiplied by the constant flow of oxygen to obtain the volume of expired nitrogen. A two point calibration is performed with known air volumes. With the amount of nitrogen washed out measured and the initial fractional alveolar nitrogen concentration known $\left(\mathrm{F}_{\mathrm{Ai}}\left(\mathrm{N}_{2}\right)\right.$ room air $\left.=0.79\right)$, the lung volume at which the washout was initiated can be calculated ${ }^{58}$ as follows:

lung volume $(\mathrm{FRC}$ or $\mathrm{RV})=$ volume $\mathrm{N}_{2}$ washed out $/ \mathrm{F}_{\mathrm{Ai}}\left(\mathrm{N}_{2}\right)$

The PPU has an operator controlled pneumatic slide valve that switches the infant to breathing $100 \%$ oxygen. The expired gas then enters a mixing chamber that is connected via a precision needle valve and a vacuum pump to a nitrogen analyser and the nitrogen concentration is integrated electronically by the PPU signal processing system. The nitrogen washout curve is displayed in real time on the computer monitor. When a $0 \%$ nitrogen concentration is displayed on the monitor, the slide valve is activated and the infant is switched back to breathing room air and $\operatorname{FRC}\left(\mathrm{N}_{2}\right)$ or $\mathrm{RV}\left(\mathrm{N}_{2}\right)$ are automatically calculated by the system.

A systematic exploration of possible sources of error when using the nitrogen washout technique was undertaken. Potential sources of error encountered, suggested, or reported included (1) changes in background oxygen, (2) the infant's peak flow exceeding the background flow, (3) a change in calibration over time,$^{5}(4)$ switching at end tidal expiration when FRC $^{57}$ was measured or leaks in the circuit, including the face mask, and in infants with airway obstruction, (5) the unanswered question of the final nitrogen concentration, and (6) length of the washout time and the minimal time interval between consecutive measurements. ${ }^{1578}$ Over 2000 in vitro washouts were performed using the calibrating syringe as a lung model. Furthermore, simulations were performed using unequal stroke volumes and different rates with the calibrating syringe to simulate periodic breathing, sighs, rapid shallow breathing, pauses to simulate apnoeas, or temporary upper airway obstruction. Of several connections, equipment warming times, and flow rates of background oxygen tested, the methodology which yielded the best results is given below.

\section{Nitrogen washout circuit}

A three way pneumatic slide valve (8540 series, $9.5 \mathrm{~mm}$ flow bore size; Hans Rudolph Inc) was used with a mouth port of $22 \mathrm{~mm}$ outer diameter $(\mathrm{OD}) \times 15 \mathrm{~mm}$ inner diameter (ID) and two smaller inlet/outlet ports of
$15 \mathrm{~mm}$ OD $\times 10.5 \mathrm{~mm}$ ID. The oxygen flow of a high precision flowmeter (Timemeter Instrument Corporation, Lancaster, Pennsylvania, USA) was accurately set by adjusting the middle of the float to the $10 \mathrm{l} / \mathrm{min}$ mark. This flow was used for all tests. The oxygen tubing (King Systems Corporation, Noblesville, Indiana, USA) was connected to the $3.18 \mathrm{~mm}$ ID end of an adapter (Hospitak Inc, Farmingdale, New York, USA), the other end of which (22 mm ID) fitted onto the $22 \mathrm{~mm}$ OD of a " $T$ " connection (" $T$ " piece; Intersurgical Inc, Cazenovia, New York, USA). A 0.5 litre collapsible breathing bag (Vital Signs Inc, Totowa, New Jersey, USA) was attached to the centre port (22 mm ID) of the "T" connection via an adapter $(22 \mathrm{~mm}$ OD/19 $\mathrm{mm}$ ID $\times$ $22 \mathrm{~mm}$ OD/17 mm ID; Baxter Healthcare Corporation, Deerfield, Illinois, USA), and the distal (third) end (22 mm ID) of the "T" connection was fitted onto an aerosol "T" adapter (22 mm OD; Hudson Respiratory Care Inc, Temecula, California, USA). The centre port (15 mm ID) of the aerosol " $T$ " adapter was inserted onto the small port (15 mm OD) of the three way slide valve situated at right angles to the mouth port. The opposite end ( $22 \mathrm{~mm}$ OD) of the aerosol "T" adapter was inserted into a distensible coupling connector $(29 \mathrm{~mm}$ OD $\times 17 \mathrm{~mm} \mathrm{ID}$; Marquest Medical, Aurora, Colorado, USA) to obtain a snug fit. A Concha Hose Adapter (Respiratory Care Inc, Arlington Heights, Illinois, USA) joined the other end of the coupling connector and the proximal end of a $2.0 \mathrm{~m}$ long hose (Tygon, $9.53 \mathrm{~mm}$ ID $\times$ $15.88 \mathrm{~mm}$ OD; Baxter Healthcare Corporation) with a snug fit. The distal end of the hose was inserted into the $11 \mathrm{~mm} O D$ end of an adapter (Marquest Medical, Aurora, Colorado, USA) whose other end (22 mm ID) fitted snugly into the inlet port of the nitrogen mixing chamber. A $1.8 \mathrm{~m}$ long (22 mm ID) corrugated tube (Baxter Healthcare Corporation) was attached to the outlet port of this chamber and was loosely coiled in an open box on the side of the PPU. This tube prevented ambient air from diffusing back into the nitrogen mixing chamber.

\section{Calibration of the PPU}

As recommended by the manufacturer, using separate electrical outlets the vacuum pump with its oxygen flow was turned on 30 minutes before calibration and the PPU measurement module and computer at least 20 minutes before calibration. The breathing bag was squeezed manually several times to wash out any nitrogen. Since the computer software automatically converts measured washout volumes to BTPS, we chose to make all measurements under ambient (ATPS) conditions by entering, before testing, a temperature of $37^{\circ} \mathrm{C}$ and a barometric pressure of $760 \mathrm{~mm} \mathrm{Hg}$ when prompted by the software program. This facilitated the comparison between integrated nitrogen signals obtained when injecting a known volume during calibration and using this same volume to confirm calibration. The calculated volume of the latter would otherwise 
have been automatically converted to BTPS. This allowed the operator to pinpoint potential sources of errors during measurements. The computer software nitrogen calibration menu was accessed. The displayed nitrogen concentration was $0.0 \%$ and, if not, it was zeroed using the "autozero" mode of the PPU software. A check for the presence of a baseline drift was then performed. The menu of the "low" (or "high") volume calibration was accessed. When the displayed nitrogen concentration was $0.0 \%$, the slide valve was activated and the mouth port was left open to room air for 10 seconds in order to wash out the air within the port with the pure oxygen. The port was then occluded to prevent room air from diffusing back into the port. The nitrogen concentration and the integrated percentage nitrogen signal were observed for 90 seconds for a stable $0.0 \%$ reading. If the integrated nitrogen signal rose, a baseline drift was presumed to be present, the "escape" key on the computer keyboard was pressed, and the calibration menu was reaccessed. Although the displayed nitrogen concentration was still $0.0 \%$, a further decrease of the baseline towards zero was performed using 1-3 keyboard strokes in the manual mode of the program, the space bar of the keyboard was then pressed to enter the baseline change, followed by a repeat check for a baseline drift. Overcorrection resulting in a negative percentage nitrogen concentration reading was not allowed. Additional checks for baseline drifts (and correction if needed) were undertaken during the waiting periods between measurements on an infant with the slide valve being activated via the "patient test" menu rather than the "calibration" menu used initially.

Calibration of the nitrogen analyser needle valve was performed before each test. The needle was removed from the outlet port of the mixing chamber and allowed to hang for at least 45 minutes so that room air was sampled. The procedure of "peaking the needle", as described by the manufacturer, was performed to obtain the optimum negative pressure in the analyser to achieve maximum nitrogen ionisation. When the needle was replaced into the outlet port the displayed nitrogen concentration was $0.0 \%$. The low and high volume calibration was performed with $42 \mathrm{ml}$ and $342 \mathrm{ml}$ air, respectively, for all infant tests. When the integrated nitrogen signal stopped rising it was recorded, the "escape" key was pressed, and each volume calibration was repeated until two successive integrated nitrogen signals within $1 \%$ of each other were obtained before the calibration was entered. The accuracy of the calibration was rechecked immediately after calibrating by measuring known volumes of air (see below). It was also rechecked after a test had been completed using a sterilised nitrogen circuit (slide valve, connectors, and a new collapsible breathing bag) to prevent contamination of the calibrating syringe. A known volume equivalent to the infant's measured lung volume was included in rechecking the calibration. Finally, the nitrogen analyser needle was removed from the outlet port of the nitrogen mixing chamber and allowed to hang for about
15 minutes for room air to be sampled. The displayed nitrogen concentration was 79.0 $(0.2) \%$ after all tests.

ACCURACY STUDY WITH A LUNG MODEL

When the 0.5 litre calibrating syringe with its piston pushed in to the $500 \mathrm{ml}$ mark was connected to the mouth port of the slide valve $(22 \mathrm{~mm}$ OD $\times 15 \mathrm{~mm}$ ID) their combined volume was found to be $42 \mathrm{ml}$. This was estimated by volume replacement and was further confirmed by Hans Rudolph Inc. This volume was not subtracted during calibration. The gas space within the slide valve piston body was ignored because it was flushed with oxygen during testing for baseline drifts. Following a two point calibration the syringe was used as a lung model to wash out known air volumes from $42 \mathrm{ml}$ to $492 \mathrm{ml}$. The background flow of oxygen was set at $10 \mathrm{l} / \mathrm{min}$. Each measurement was repeated five times. Using stroke volumes of $50-75 \mathrm{ml}$, different known air volumes were randomly used over a 14 hour period to determine whether changes in calibration occurred over time.

Introduction of the collapsible breathing bag into the oxygen circuit enhanced the reproducibility and precision of volume measurements. Therefore, using stroke volumes of approximately $70 \mathrm{ml}$ to simulate tidal breathing, as was routinely done for calibration, the influence of the breathing bag was studied by comparing the waveform of the flow and volume signals before and after introducing the breathing bag into the circuit. This in vitro experiment was performed by inserting the PNT into the distal end of the corrugated tube, the proximal end of which was connected to the outlet port of the nitrogen mixing chamber.

\section{STATISTICAL ANALYSIS}

Data were expressed as arithmetic mean and standard deviation (SD). The Student's $t$ test for paired data was used to analyse data from the accuracy study to compare known volumes with volumes measured by the nitrogen washout technique. $p$ values of less than 0.05 were considered significant.

\section{Results}

ACCURACY STUDY WITH THE LUNG MODEL

Results from this in vitro study are presented in table 1 . The mean coefficient of variation $(\mathrm{CV})$ of all volumes was $0.66 \%$. The mean difference

Table 1 Accuracy study: measurement of known air volumes by nitrogen washout

\begin{tabular}{lll}
\hline $\begin{array}{l}\text { Known volume } \\
(\mathrm{ml})\end{array}$ & $\begin{array}{l}\text { Measured volume } \\
(\mathrm{ml})\end{array}$ & Error (\%) \\
\hline 42 & $41.8(0.4) \dagger$ & -0.47 \\
92 & $92.2(0.6)$ & 0.22 \\
142 & $141.9(1.1)$ & -0.07 \\
192 & $191.1(1.2)$ & 0.46 \\
242 & $242.4(1.9)$ & -0.16 \\
292 & $290.9(1.4)$ & -0.37 \\
342 & $341.6(1.8)$ & -0.11 \\
392 & $391.9(2.6)$ & -0.02 \\
442 & $442.8(2.9)$ & -0.18 \\
492 & $490.4(1.4)$ & -0.32 \\
& & $-0.10(0.28)^{\star}$ \\
\hline
\end{tabular}

${ }^{\star}$ Mean (SD).

tValues are mean (SD) of five measurements performed randomly over a 14 hour period. 

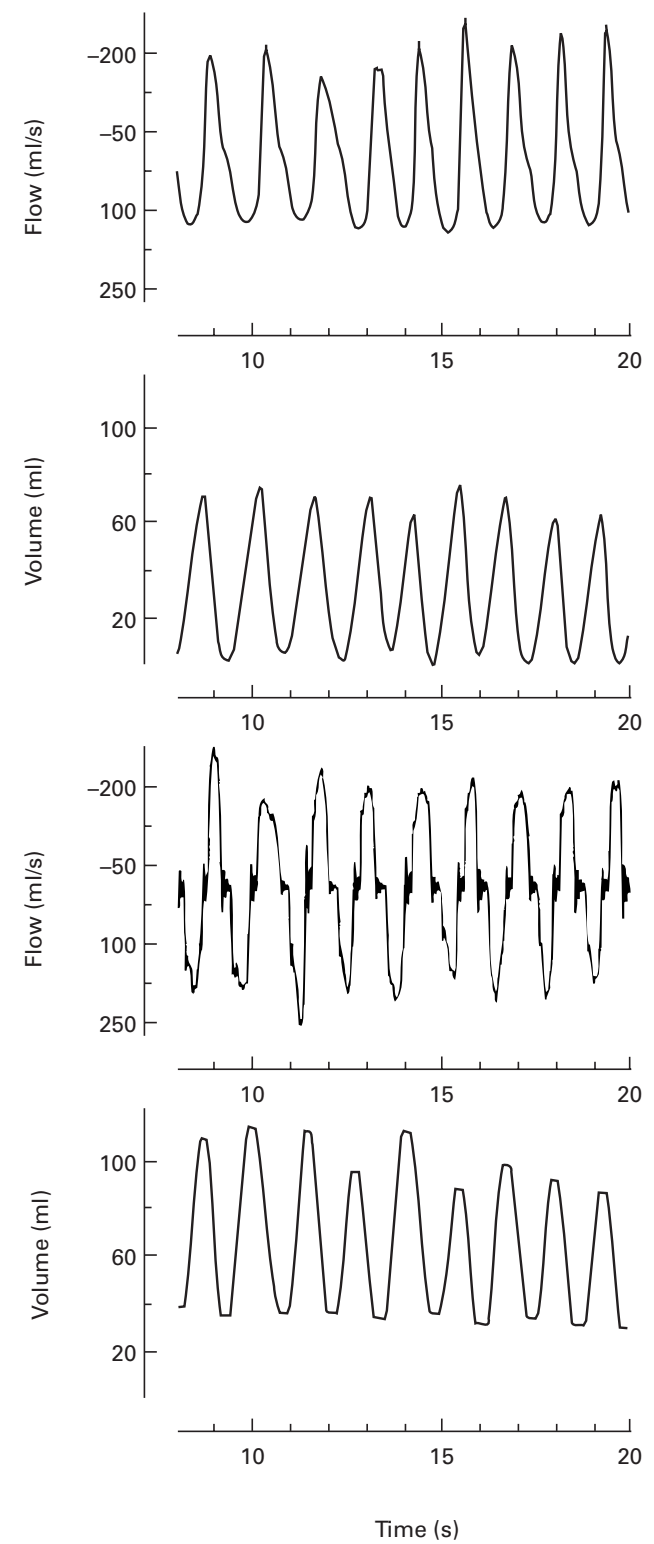

Figure 1 Influence of the collapsible breathing bag on gas flow in the nitrogen mixing chamber during in vitro washout. Using stroke volumes from the calibrating syringe of about $70 \mathrm{ml}$ to simulate tidal breathing, the flow and volume signals pattern were compared using a

pneumotachometer connected to the outlet of the nitrogen mixing chamber before (lower two panels) and after (upper panels) the breathing bag was introduced into the washout circuit. Note the presence of flow transients in the absence of the breathing bag.

between measured and known volumes was $0.30 \mathrm{ml} \quad(95 \% \quad \mathrm{CI}-0.18$ to 0.79$)$. This difference was not statistically significant $(\mathrm{p}=$ $0.22)$. The mean percentage error was $-0.1 \%$ (range $-0.47 \%$ to $0.46 \%$ ).

The breathing bag had a clear influence on the waveform of the flow and volume signals in vitro. In the absence of the breathing bag, flow transients were frequent (fig 1).

\section{Discussion}

Of several connections, equipment warming times, and magnitudes of background oxygen flow, the methodology described here gave the most reproducible results in vitro and in vivo. ${ }^{7}$ Calibration remained stable during 14 hours of in vitro testing (table 1 ) as well as after infant testing had been completed. ${ }^{7}$ The nitrogen washout circuit can be quickly assembled into a virtually leak-free unit that can be disinfected between tests.

Inclusion of the collapsible breathing bag in the nitrogen washout circuit had a significant stabilising effect that led to exceptional reproducibility in vivo ${ }^{7}$ and accuracy in vitro (table 1) in measured washout volumes. The in vitro study indicated that flow transients were more frequent in the absence of the breathing bag (fig 1). To ensure that the observed waveform difference was not the result of a technical variation in injecting air from the calibrating syringe, tidal breathing indices (tidal volume, the inspiratory, expiratory and total time and frequency) were computed from the in vitro study. Using the unpaired $t$ test, no significant difference was found between the mean values of the indices before and after the breathing bag was placed in the circuit (data not shown). Given the low resistance in the open circuit of nitrogen washout, the breathing bag probably acted as a buffer reservoir that prevented large swings in the bias flow of oxygen in the nitrogen mixing chamber. These swings inevitably occur during the breathing cycle of an older child with a larger tidal volume. More importantly, the breathing bag minimised the retrograde movement of mixed oxygen and nitrogen after it had passed beyond the sampling needle port. This explanation was supported by the observation that, when a tube shorter than the $1.8 \mathrm{~m}$ tube used in our study was attached to the outlet port of the nitrogen mixing chamber, a sharp rise in the nitrogen washout curve above baseline occurred when an older child took a deep breath. This was presumably due to the retrograde movement of gas enriched with room air nitrogen into the outlet port and through the sampling needle which is located at a depth of $30 \mathrm{~mm}$ from the port opening (15 mm ID)-that is, separated from the opening by only $5.3 \mathrm{~cm}^{3}$. An in vitro simulation using a sharp pull on the piston of the calibrating syringe lends support to this hypothesis. The breathing bag served also as a constant monitor for tidal breathing. In the author's experience, an occasional transient upper airway obstruction in the sedated infant was easily detected and promptly corrected during washouts. Pauses during periodic breathing were also noted. If either of these two phenomena were to occur close to the end of a washout, they could conceivably lead to a premature return of the nitrogen washout curve to baseline and termination of the test by the operator resulting in an underestimation of the measured washout volume. Interestingly, the detection of a transient airway obstruction during FRC measurement of an infant was slightly delayed, resulting in the return of the nitrogen washout curve to baseline. The infant's head was repositioned which relieved the obstruction, which in turn was confirmed by the breathing bag. The nitrogen curve rose again above baseline when the washout resumed. This produced the second peak of what became a biphasic curve whose calculated 
volume was still reliable. An in vitro simulation with the calibrating syringe performed by pausing during washout resulted in a biphasic curve that reliably reflected the known volume (M G Morris, personal observation). Indeed, it would be reasonable not to terminate an infant test immediately when a zero nitrogen concentration is displayed on the monitor unless the breathing bag is still synchronously inflating and deflating with the infant chest wall excursions. A substantial or nearly complete deflation of the breathing bag during a washout by the inspiring child could presumably alert the operator to the possibility of a peak inspiratory flow exceeding the bias flow of oxygen. The insertion of a pneumotachometer between the face mask and the mouth port of the slide valve could provide alternative continuous monitoring for tidal breathing during washouts. ${ }^{8}$ However, this has the disadvantage of increasing the dead space and the resistance of the washout circuit without providing the buffering capability of the breathing bag for large tidal volumes. Taken together, we conclude that the breathing bag should be an integral component of the open nitrogen washout circuit. The optimal location of the breathing bag in the circuit was between the patient and the oxygen source, and closer to the former. Placement distal to the patient tended to retain some of the washed out nitrogen and, unless the bag was slowly and completely squeezed just before the washout was completed, measured volumes would be underestimated (M G Morris, unpublished data).

Adequate equipment warming time was an important factor in performing reliable nitrogen washouts. Hanging the needle valve for at least 45 minutes to sample room air was found, by using longer periods up to two hours, to be essential to achieve a stable nitrogen concentration reading in our PPU. Premature termination of this step contributed to the change in nitrogen calibration over time and with the finding, after infant testing had been completed, of a nitrogen concentration below $79 \%$ when the needle was used to resample room air.

Drifting of the baseline has been a major challenge during testing and a potential source for a systematic error by overestimating measured washout volumes. Frequent checks for baseline drifts and correction were therefore essential to obtain reproducible data. Interestingly, drifting might not occur for 60 seconds but then the integrated nitrogen signal would start rising rapidly. The author has observed a stability of the baseline lasting up to 15 minutes. Hence, in testing patients with airway obstruction it is important to test for drifts for at least 90 seconds because of the expected prolonged washout times. ${ }^{7}$ An immediate rise above baseline of the nitrogen washout curve after the slide valve was switched indicated the presence of a drift if air had not yet entered the bias flow of oxygen from either the calibrating syringe or the apnoeic infant during measurement of RV. ${ }^{7}$ A true rise in the integrated nitrogen signal was found when a tube shorter than the $1.8 \mathrm{~m}$ corrugated tube used in our study was attached to the mixing chamber outlet port, allowing room air to diffuse back into the port and sampling needle. Another potential source was a discontinuity in any of the connections, and this happened only once through a visible break in the aerosol " $T$ " connection port.

Using the calibrating syringe as a lung model, the author has used a wide range of volumes for several two point calibration experiments. These in vitro experiments suggested that the system remained linear for at least $50 \mathrm{ml}$ beyond the two point calibration. We chose $42 \mathrm{ml}$ and $342 \mathrm{ml}$ for all infant tests ${ }^{7}$ so that we would become familiar with their respective integrated nitrogen signal and easily detect aberrant numbers. This added a quality assurance element to our operating conditions. The use of a small volume $(42 \mathrm{ml})$ has the advantage of shortening the calibration time. After an infant test had been completed, a known volume equivalent to the patient's measured lung volume was routinely included in re-checking the calibration.

Inadequate equilibration of the calibrating syringe with room air nitrogen after it had been used for calibration or to confirm calibration seemed a real and frequent, but avoidable, source of error by underestimating "presumed" known volumes. This was likely to occur when a large volume such as $492 \mathrm{ml}$ had been used by the hurried operator. We used a 15 litre flow of air via a cannula inserted for a brief period into the calibration syringe assisted by a few piston strokes to speed up the equilibration process. After the cannula had been pulled out, a few more piston strokes were used for a final equilibration with room air. This was preferable to loosening the volume adjustment metal ring to achieve larger excursions of the piston in order to preserve the exact known volume for a repeat injection. Similarly, the mouth port of the slide valve should be flushed with room air after a washout. After a washout had been completed and the slide valve had switched the calibrating syringe to room air, the piston was routinely pulled back before disconnecting the syringe from the mouth port to flush the latter with room air.

Since clinical settings are usually associated with time constraints, the calibration of the PPU described here seemed adaptable for use in these settings. ${ }^{7}$ Whereas the manufacturer recommended warming periods of 30 minutes and 20 minutes for the nitrogen pump and computer module, respectively, there was no mention of a time period being required for the nitrogen analyser needle to equilibrate with ambient nitrogen. Similarly, there was no information regarding baseline drifting. These were discovered during our in vitro studies. A 90-100 minute calibration period was usually sufficient for the PPU to be ready for clinical testing. This included 30 minutes for pump and computer module, 45 minutes for the sampling needle equilibration with room air nitrogen, "peaking the needle", testing for baseline drifts, the two point calibration (see above), and confirmation of calibration immediately after calibration with at least two known 
volumes. The ultimate result, however, is a one day stable calibration that allows testing of more than one infant per day. Moreover, the described methodology rewards the operator with excellent repeatability of measurements such that fewer reliable tests need to be performed on a sedated infant. ${ }^{7}$

In conclusion, we developed a method that increased the accurancy in vitro and the precision in vivo ${ }^{7}$ of volume measurement using the open circuit nitrogen washout technique. Sources of potential errors that included baseline drifting and inadequate equipment warming times were evaluated. We conclude that a collapsible breathing bag should be incorporated in the nitrogen washout circuit.

Supported by a Clinical Research Grant (CG-008-N) cofunded by the American Lung Association (ALA) and the Arkansas Chapter of ALA. The author was supported in part by the Department of Pediatrics, UAMS.
1 American Thoracic Society/European Respiratory Society. Respiratory mechanics in infants: physiological evaluation in health and disease. Am Rev Respir Dis 1993;147:474-96. 2 Gaultier C. Lung volume in neonates and infants. Eur Respir f 1989;2(Suppl 4):130-4s.

3 Gerhardt T, Hehre D, Bancalari E, et al. A simple method for measuring functional residual capacity by nitrogen washout in animals and newborn infants. Pediatr Res 1986; 20:668-71.

4 Sivan Y, Deakers TW, Newth CJL. An automated bedside method for measuring functional residual capacity by method for measuring functional residual capacity by
nitrogen washout in mechanically ventilated children. Pedinitrogen washout in mechar

5 Gappa M, Fletcher ME, Dezateux CA, et al. Comparison of nitrogen washout and plethysmographic measurements of lung volume in healthy infants. Am Rev Respir Dis 1993;148:1496-501. 6 Eber E, Steinbrugger B, Modl M, et al. Lung volume meas-
urements in wheezy infants: comparison of plethysmography and gas dilution. Eur Respir f 1994;7:1988-94.

7 Morris MG. A novel noninvasive technique for measuring the residual lung volume by nitrogen washout with rapid thoracoabdominal compression in infants. Thorax 1999 (in press).

8 Tepper RS, Merth IT, Newth CJL, et al. Measurement of functional residual capacity in infants by helium dilution and nitrogen washout techniques. In: Stocks J, Sly PD, Tepper RS, Morgan WJ, eds. Infant respiratory function testing. New York: John Wiley \& Sons Inc, 1996: 165-89. 